\title{
MicroRNA-144 inhibits migration and proliferation in rectal cancer by downregulating ROCK-1
}

\author{
SHANG-DANG CAI ${ }^{1 *}$, JIAN-SHE CHEN $^{2 *}$, ZUO-WU XI $^{1}$, LONG-JIANG ZHANG ${ }^{1}$, \\ MING-LIAO NIU ${ }^{1}$ and ZONG-YUE GAO ${ }^{1}$ \\ ${ }^{1}$ Anorectal Branch and ${ }^{2}$ Center for Reproductive Medicine, Henan Province Hospital of TCM, \\ Zhengzhou, Henan 450002, P.R. China
}

Received December 19, 2014; Accepted September 10, 2015

DOI: $10.3892 / \mathrm{mmr} .2015 .4391$

\begin{abstract}
Cancer of the colon and rectum are two distinct entities, which require different treatment strategies and separate treatment. MicroRNAs (miRNAs) act as critical regulators of genes involved in several biological processes. Aberrant alterations of miRNAs have been found in several types of cancer, including colon cancer and rectal cancer. Extensive catalogues of downregulated miRNAs have been identified for colon cancer, whereas only limited data are available for rectal cancer. An example of miRNA profiling in a previous study found that miRNA (miR)-144 showed aberrant expression and appeared to be rectal cancer-specific, its expression not being reported in colon cancer. In the present study, the role of miR-144 in rectal cancer was investigated. SW837 and SW1463 cell lines were selected as rectal cell carcinoma cells. Using reverse transcription-quantitative polymerase chain reaction, western blot, BrdU, cell migration and cell viability assays, it was found that the expression levels of miR-144 were significantly reduced in the SW837 and SW1463 cell lines, and the overexpression of miR-144 suppressed rectal cancer cell viability, migration and proliferation. In addition, Rho-associated coiled-coil containing protein kinase 1 (ROCK1) was identified as a target of miR-144 in the rectal cancer cells. The supplementation of ROCK1 markedly restored the cell migration and proliferation, which was
\end{abstract}

Correspondence to: Dr Shang-Dang Cai, Anorectal Branch, Henan Province Hospital of TCM, 6 Dongfeng Road, Zhengzhou, Henan 450002, P.R. China

E-mail: shangdangcai@163.com

*Contributed equally

Abbreviations: miR-144, microRNA-144; ROCK1, Rho-associated coiled-coil containing protein kinase 1; CRC, colorectal cancer; miRNAs, microRNAs; E2F3, E2F transcription factor 3; ZEB1, zinc finger $\mathrm{E}$ box binding homeobox 1; ZEB2, zinc finger $\mathrm{E}$ box binding homeobox 2

Key words: microRNA-144, rectal cancer, Rho-associated coiled-coil containing protein kinase 1 inhibited by miR-144. Together, the data of the present study demonstrated that miR-144 acts as a tumor suppressor by targeting ROCK1, and indicates the potential of miR-144 as a novel biomarker and target in the treatment of rectal cancer.

\section{Introduction}

A class of small non-coding RNAs, termed miRNAs are now recognized, which contribute to essential biological processes, including development, cellular differentiation, proliferation, stress responses, apoptosis and metabolism, as well as tumor initiation and progression $(1,2)$. These miRNAs are 18-24 nucleotides long (3). miRNAs are considered to exhibit tumor suppressor gene and oncogene regulatory roles, and show complex patterns of disease-and tissue-specific expression, which are associated with their ability to regulate several targets that are crucial to the carcinogenic process (2). In multiple types of cancer, aberrant patterns of miRNA expression have been observed, which can affect cancer cell proliferation (4), apoptosis (5) and metastasis (6), and potentially define the cancer stem cell phenotype (7).

Colorectal cancer (CRC), the third most common type of cancer worldwide, is the fourth leading cause of cancer-associated mortality, accounting for $90 \%$ incidence and mortality rates $(8,9)$. In 2003 , the differential expression of miRNAs was first reported to be associated with CRC (10). From a clinical point of view, cancer of the colon and rectum are two distinct entities, which require different treatment strategies $(2,11)$. Accordingly, they require separate treatment when analyzing the genetics and biology of the diseases. For colon cancer, extensive catalogues of downregulated miRNAs have been identified in the previous years, whereas only limited data are available for rectal cancer (2).

In order to understand the function of miRNA in human rectal cancer, miRNA profiling was performed in a previous study (1), wherein microRNA-144 was found to show aberrant expression and was identified as rectal cancer-specific; having not been reported in colon cancer. Originally, miRNA (miR)-144 was identified as an erythroid-specific miRNA, which is required for subsequent erythroid lineage survival and maturation $(12,13)$. In addition, it can increase the severity of anemia, and decrease glutathione regeneration and antioxidant capacity (14). Previous tumor investigations, involving a 
comprehensive meta-analysis of miRNA expression microarrays, revealed that miR-144 is downregulated in lung cancer, prostate cancer and hepatocellular carcinoma (15), indicating that it may be a novel human cancer-associated miRNA. To date, there are no reports that miR-144 has a functional role in rectal cancer. In the present study, miR-144 was characterized in rectal cancer and found that the upregulation of miR-144 inhibited malignant progression (migration and proliferation) of rectal cancer cells. According to previous reports, Rho-kinase (ROCK) is a serine/threonine kinase, which functions downstream of the small GTPase RhoA (16). ROCK isoforms have been implicated in a variety of cellular functions, including smooth muscle contraction, actin cytoskeleton organization (17), cytokinesis (18), cell adhesion and motility (19). In addition, the activation of ROCK1 by RhoA can promote cell invasion and motility in prostate cancer and colorectal carcinoma cells $(20,21)$. In order to determine whether the inhibitory effects of miR-144 on the progression of rectal cancer are associated with ROCKs, the present study performed a series of relative experiments, and found that miR-144 inhibits the migration and proliferation of SW837 and SW1463 rectal cancer cells by targeting the crucial oncogene, ROCK1.

\section{Materials and methods}

Cell lines and cell culture. Human SW837 and SW1463 cell lines were purchased from American Type Culture Collection (ATCC; Manassas, VA, USA). These two cell lines originated from rectum tissue belonging to epithelial cells, and they were selected as rectal cell carcinoma cells. The SW837 and SW1463 cells were cultured in Leibovitz's 15 (L-15) culture medium (cat. no. 30-2008; ATCC) supplemented with fetal bovine serum (Sigma-Aldrich, St. Louis, MO, USA), to a final concentration of $10 \%$ at $37^{\circ} \mathrm{C}$. The cells were passaged $1-2$ times per week and were cultured in free gas exchange with atmospheric air. A human rectal mucosa epithelium cell was purchased from PriCells (Wuhan, China); which was derived from normal rectal mucosa epithelium and was used as a normal control (NC).

Reverse transcription-quantitative polymerase chain reaction (RT-qPCR) analysis. Total RNA and miR were extracted from the above cell line $\left(5 \times 10^{5}\right.$ per well in six-well plates at $80 \%$ confluency) using an RNeasy Mini kit and an miR Neasy Mini kit (Qiagen, Valencia, CA, USA). Following quantitation, the extracted total RNA was reverse-transcribed using a High Capacity cDNA Archive kit (Applied Biosystems; Thermo Fisher Scientific, Inc., Waltham, MA, USA) and a TaqMan microRNA reverse transcription kit (Applied Biosystems), according to the manufacturer's protocol. The RT products were mixed with TaqMan universal PCR master mix II, and qPCR was performed on an Applied Biosystems Prism 7500 Fast Sequence Detection System (Applied Biosystems; Thermo Fisher Scientific, Inc.). The PCR parameters were as follows: $95^{\circ} \mathrm{C}$ for $20 \mathrm{sec}, 40$ cycles at $95^{\circ} \mathrm{C}$ for $3 \mathrm{sec}$ and $60^{\circ} \mathrm{C}$ for $30 \mathrm{sec}$. The primers for mRNA were as follows: miR-144 (cat. no. 204754; Exiqon A/S, Vedbaek, Denmark); ROCK-1, forward 5'-AAG AGA GTG ATA TTG AGC AGT TGC G-3' and reverse 5'-TTC CTC TAT TTG GTA CAG AAA GCC A-3'; and ROCK2, forward 5'-TGC GGT CAC AAC TCC AAG C-3' and reverse 5'-GGA AAC CCA TCA TCT
GCC TCA G-3'. RNU48 or $\beta$-actin were used as an endogenous control. Primers were synthesized by Shanghai Sangon Biological Engineering and Technology Service (Shanghai, China). All reactions were performed in triplicate. The mRNA and miRNA expression levels were determined using the $2^{-\Delta \mathrm{Cq}}$ method (22).

Cell transfection and immunoblotting. The SW837 and SW1463 cells $\left(5 \times 10^{5}\right.$ per well in six-well plates, $80 \%$ confluency) were transfected with pre miR-144 or a scrambled pre miR-control (Ambion; Thermo Fisher Scientific, Inc.) using Lipofectamine 2000 (Invitrogen; Thermo Fisher Scientific, Inc.), according to the manufacturer's protocol. Following transfection for $72 \mathrm{~h}$, for miR-144 or mRNA (ROCK1 and ROCK2) analysis, total RNA was extracted using previously described methods (23). The cells were lysed in Triton X-100 containing $1 \%$ phosphate-buffered saline (PBS) with a cocktail of protease inhibitors (Roche Diagnostics, Basal, Switzerland) for protein analysis. The lysates were then centrifuged at $16,000 \mathrm{~g}$ for $15 \mathrm{~min}$, and the protein concentrations of the supernatants were determined using a bicinchoninic acid assay kit (Pierce Biotechnology, Inc., Rockford, IL, USA). The ROCK1 and ROCK2 proteins (40 $\mu \mathrm{g} /$ lane) were analyzed using immunoblotting following transfer onto polyvinylidene fluoride membranes (Sigma-Aldrich) from a 10\% SDS-PAGE gel (Sigma-Aldrich). Subsequently, followingblocking with $0.5 \%$ skimmed milk powder (Sigma-Aldrich) in $1 \mathrm{X}$ PBS-Tween 20 (Sigma-Aldrich), the membranes were incubated with primary monoclonal rabbit anti-human ROCK1 antibody (cat. no. ab45171) or monoclonal rabbit anti-human ROCK2 antibody (cat. no. ab125025), and monoclonal rabbit anti-human $\beta$-actin antibody (cat. no. ab115777), at a dilution of $1: 4,000$ overnight at $4^{\circ} \mathrm{C}$. Following washing three times (10 min each) with $1 \mathrm{X}$ PBS-Tween 20 , the membranes were incubated with horseradish peroxidase (HRP)-conjugated polyclonal goat anti-rabbit IgG (cat. no. ab6721) secondary antibody $(1: 10,000)$ at room temperature for $1 \mathrm{~h}$, and washed again. The reactive bands were detected using enhanced chemiluminescence (GE Healthcare Life Sciences, Chalfont, $\mathrm{UK}$ ), according to the manufacturer's protocol. The relative levels of each protein to $\beta$-actin were analyzed. All primary and secondary antibodies were purchased from Abcam (Cambridge, MA, USA). The resulting bands of the blot were analyzed using Gel-Pro Analyzer 4.0 software (Media Cybernetics Inc., Silver Spring, MD, USA).

Cell viability. Following transfection with pre-miR-144, the viabilities of the SW837 or SW1463 cells were determined using an MTT assay (Sigma-Aldrich). Briefly, $5 \times 10^{3}$ transfected cells were plated in 96 -well plates. Following incubation at $37^{\circ} \mathrm{C}$ for different periods of time (24, 48 and $72 \mathrm{~h})$, the culture medium was removed and MTT $(10 \mu \mathrm{l} ; 0.5 \%)$ was added. Following incubation for another $4 \mathrm{~h}$ at $37^{\circ} \mathrm{C}$, the culture medium was replaced with dimethyl sulfoxide (10 $\mu \mathrm{l}$; 4\%; Sigma-Aldrich), and the optical density (O)570 was measured using a microplate reader (Multiskan MK2; Molecular Devices; Thermo Fisher Scientific, Inc.).

Cell migration analysis using a Transwell assay. Migration assays were performed in Transwells (8.0- $\mu \mathrm{m}$ pore size). 
For the migration assay, the miR-144-transfected SW837 and SW1463 cells $\left(\sim 2 \times 10^{4}\right.$ cells per well), in L-15 culture medium containing $10 \%$ FBS, were added to the upper wells. L-15 media, containing $10 \%$ FBS was added to the lower wells. The miR-control-transfected SW837 and SW1463 cells were used as a control. The cells, which migrated through the filter after 24 , 48 or $72 \mathrm{~h}$ were stained with $0.1 \%$ crystal violet (Sigma-Aldrich) and counted using phase contrast microscopy (Axiovert 200M; Carl Zeiss, Jena, Germany) (24). The same experiments were performed to detect the migration abilities of the miR-144 and ROCK1 co-transfected SW837 and SW1463 cells.

Bromodeoxyuridine (BrdU) assay. A BrdU Cell Proliferation Assay kit (cat. no. 2752; EMD Millipore, Bedford, MA, USA) was used to detect cell proliferation ability. The miR-144-transfected SW837 and SW1463 cells were synchronized and plated in 96 wells $\left(3 \times 10^{3}\right.$ cells/well) in L-15 culture medium containing $10 \% \mathrm{FBS}$, following which $10 \mu \mathrm{l} \mathrm{BrdU}$ solution was added and the cells were incubated at $37^{\circ} \mathrm{C}$ for $24 \mathrm{~h}, 48 \mathrm{~h}$ and $72 \mathrm{~h}$, respectively. Following incubation, $100 \mu \mathrm{l} /$ well fixing solution $(2752 \mathrm{~b}$; $4 \%$ paraformaldehyde in PBS; EMD Millipore) was added and the cells were incubated at $37^{\circ} \mathrm{C}$ for $15 \mathrm{~min}$. Subsequently, $100 \mu \mathrm{l} /$ well of pre-prepared detection antibody solution (anti-BrdU mouse monoclonal antibody; 2752c) was added and incubated at $37^{\circ} \mathrm{C}$ for $1 \mathrm{~h}$, following which $50 \mathrm{X}$ plate wash concentrate (2752h) was diluted and used to wash the plates. Subsequently, $100 \mu 1 /$ well of prepared HRP-conjugated goat anti-mouse IgG secondary antibody (2752e) was added and incubated at $37^{\circ}$ for $30 \mathrm{~min}$. The plates were washed with diluted plate wash concentrate. Finally, $100 \mu$ TMB substrate was added prior to incubation for $30 \mathrm{~min}$. The quantity of $\mathrm{BrdU}$, which was incorporated into the cells was determined at $450 \mathrm{~nm}$ using a microplate reader. The same experiments were performed to detect the proliferation ability of the miR-144 and ROCK1 co-transfected SW837 or SW1463 cells.

miR-144 and ROCK1 co-transfection assays. The SW837 and SW1463 cells were first transfected with pre miR-144 using Lipofectamine 2000, according to the manufacturer's protocol. Following transfection, the cDNA of ROCK1 was sub-cloned using pyrobest DNA polymerase (Takara Bio, Inc. Otsu, Japan) and inserted into a pEGFP-N1 vector (Clontech Laboratories, Inc., Mountain View, CA, USA). These vectors were transfected into the miR-144-transfected SW837 and SW1463 cells using Lipofectamine 2000. Mock-pEGFP-N1 (mock-ROCK1) was used as a control. The cells were used for subsequent experiments following transfection for 24,48 or $72 \mathrm{~h}$.

Statistical analysis. Each data point was obtained from three repeated experiments. Data are expressed as the mean \pm standard deviation, and differences were analyzed using Student's t-test. $\mathrm{P}<0.05$ was considered to indicate a statistically significant difference. The western blotting experiments were performed several times with similar results, of which the optimal image was selected to present.

\section{Results}

Expression of miR-144 in rectal carcinoma cell lines. To identify miR-144 in rectal carcinoma, SW837 and SW1463 cell
A
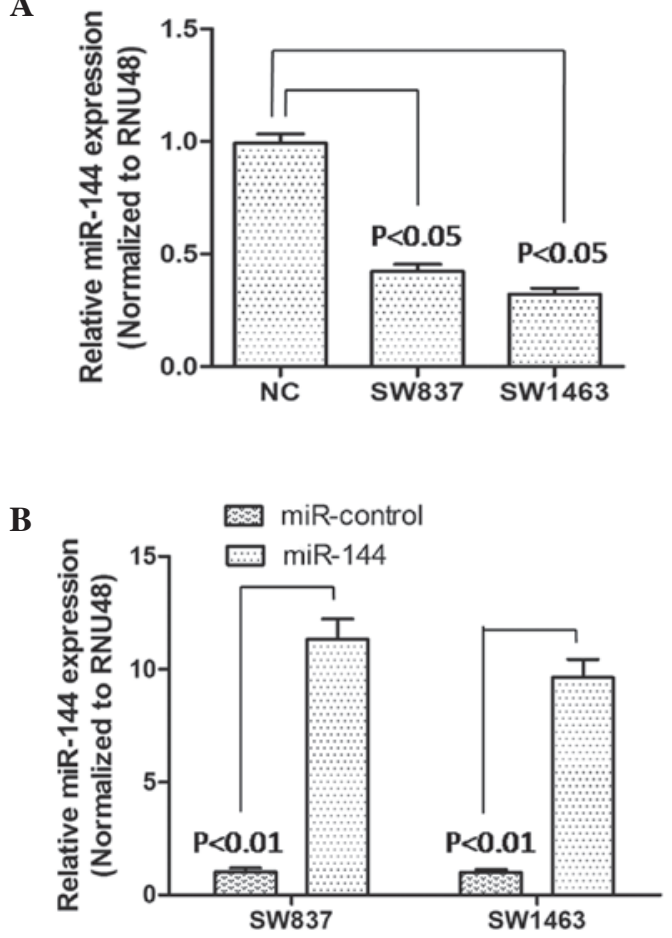

Figure 1. Expression of miR-144 in rectal carcinoma cell lines. (A) NC and rectal carcinoma cell lines (SW837 and SW1463). The expression levels of miR-144 were normalized to RNU48. Data are presented as the mean \pm standard deviation of three independent experiments, and compared with the level of miR-144 in NC cells (normalized as 1). (B) Expression levels of miR-144 were determined using reverse transcription-quantitative polymerase chain reaction $72 \mathrm{~h}$ following transfection. Data are presented as the mean \pm standard deviation of three independent experiments, and compared with the expression level of miR-144 in the miR control-transfected cells (normalized as 1). miR, microRNA; NC, normal control.

lines were selected as model cells, and the expression levels of miR-144 were determined in these cells. Following RT-qPCR analysis, the results showed that the expression of miR-144 was significantly downregulated $(\mathrm{P}<0.05)$, compared with that of the NC cells (Fig. 1A). Following this, the effect of miR-144 on rectal carcinoma cells was examined by constructing miR-144-overexpressing SW837 and SW1463 cells. The transfection effectiveness was verified using RT-qPCR. As shown in Fig. 1B, the results suggested that the expression levels of miR-144 were significantly higher than those of the miR-control-transfected SW837 and SW1463 cells, which indicated that the miR-144-overexpressing SW837 and SW1463 cells constructed were suitable for subsequent experiments.

Cell viability, migration and proliferation in miR-144-overexpressing rectal carcinoma cells. Subsequently, the viability, migration and proliferation abilities of the SW837 and SW1463 cells were detected using the methods, described above. As shown in Fig. 2A, the percentage cell viability was downregulated $(\mathrm{P}<0.05)$ in the miR-144-SW837 and the miR-144-SW1463 cells, compared with the same cells transfected with the miR-control. As shown in Fig. 2B, the number of migrating SW837 and SW1463 cells increased with increasing duration $(\mathrm{P}<0.05 ; 24 \mathrm{~h}, 48 \mathrm{~h}$ and $72 \mathrm{~h})$. However, the numbers of migrated miR-144-SW837 (blue line) or miR-144-SW1463 cells (red line), were lower, compared with 
A

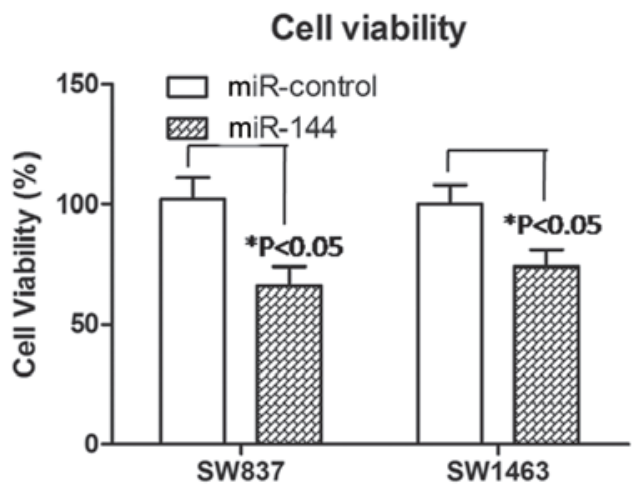

C

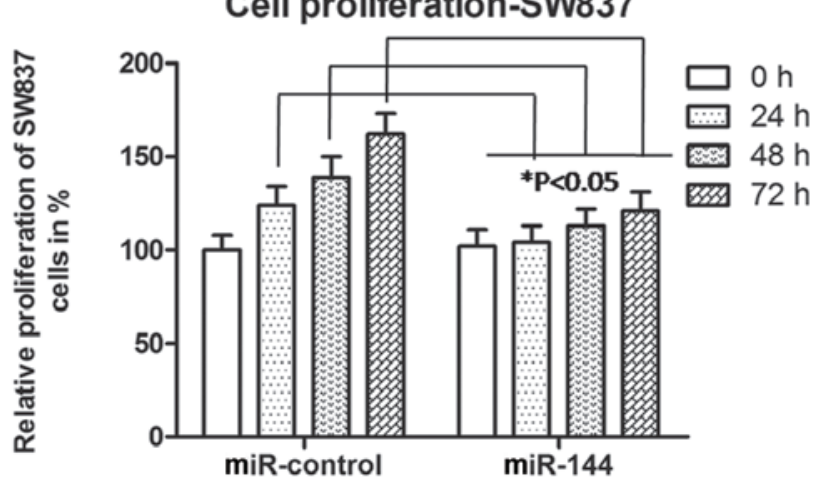

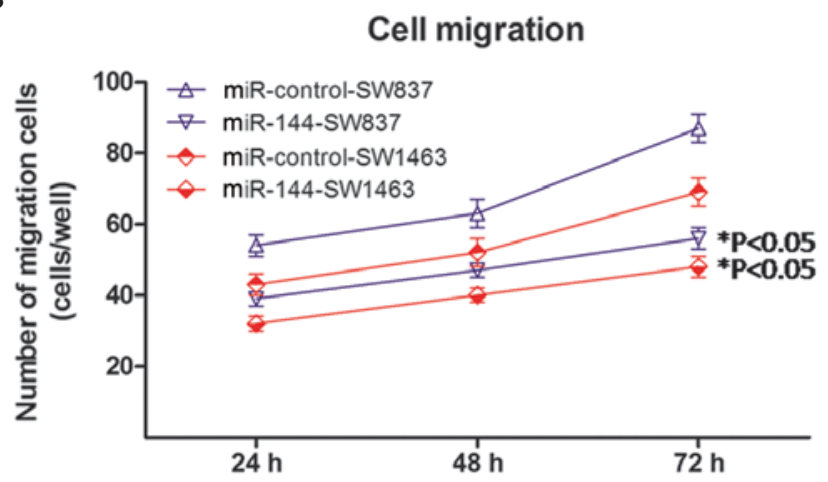

D

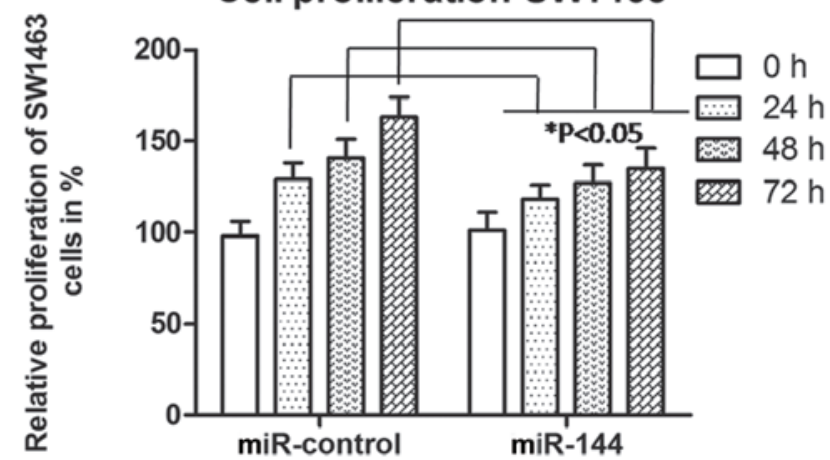

Figure 2. Cell viability, migration and proliferation in miR-144 overexpressed rectal carcinoma cells (SW837 and SW1463). (A) Cell viability was analyzed using an MTT assay following transient transfection for different durations. ${ }^{*} \mathrm{P}<0.05$, compared with the corresponding miR-control (Student's t-test). (B) Numbers of SW837 (blue lines) and SW1463 (red lines) cells, which migrated into the lower wells. Data were obtained 24 , 48 and 72 h following transfection. "P $<0.05$, compared with the corresponding miR-control group (Student's t-test). (C) Relative percentage proliferation of the SW837 cells percent. (D) Relative proliferation of SW1463 cells. Data were obtained 0, 24,48 and $72 \mathrm{~h}$ following transfection,. ${ }^{*} \mathrm{P}<0.05$, compared with the miR-control group at the corresponding time point. The data are presented as the mean \pm standard deviation of three independent experiments. miR, microRNA.

those in the miR-control-SW837 or miR-control-SW1463 groups at each time point. As shown in Fig. 2C, the relative proliferation of the miR-144-SW837 cells was lower than that of the miR-control-SW837 cells following culture for 24, 48 and $72 \mathrm{~h}$. For the proliferation of the SW1463 cells, similar results were observed (Fig. 2D). In addition, from these four experiments, the decreases in cell viability, migration and proliferation abilities was more marked in the miR-144-SW837 cells, compared with the miR-144-SW1463 cells, which may be associated with the primary levels of miR-144 in the corresponding cells. Taken together, these results in the SW837 and SW1463 cells indicated that miR-144 was involved in inhibiting the progression of rectal carcinoma.

Expression levels of ROCK1 and ROCK2 in miR-144-overexpressing rectal carcinoma cells. According to the above results, miR-144 inhibited SW837 and SW1463 cell proliferation and migration. In order to examine the underlying mechanism, the present study examined the effects of miR-144 on ROCK1 and ROCK2 in the miR-144-SW837 and miR-144-SW1463 cells. The mRNA and protein expression levels of ROCK1 and ROCK2 were detected using RT-qPCR and western blot analyses, respectively. The bands of the western blot were analyzed using Gel-Pro Analyzer 4.0 software. As shown in
Fig. 3A and B, the mRNA expression level of ROCK1 was significantly downregulated $(\mathrm{P}<0.01)$ in the miR-144-transfected cells, compared with the miR-control-transfected cells, in the SW837 and SW1463 cells. However no change in the mRNA levels of ROCK2 were observed. Similar changes were observed in the protein expression levels of ROCK1 in the SW837 cells $(\mathrm{P}<0.01)$ and SW1463 cells $(\mathrm{P}<0.05)$ and ROCK2 (Fig. 3C and D). These results suggested that the expression of ROCK1 was reduced by the overexpression of miR-144 in the SW837 and SW1463 cells.

Cell migration and proliferation in miR-144 and ROCK1-co-transfected rectal carcinoma cells. As the results revealed that only the expression of ROCK1 was affected by miR-144, co-transfection experiments were performed to further confirm the role of ROCK1, as described above. Subsequently, the cell migration and proliferation abilities of the cells were detected again by corresponding methods, described above. As shown in Fig. 4A and B, the numbers of migrated miR-144 and ROCK1 co-transfected SW837 (blue line) and SW1463 cells (red lines) were higher, compared with those of the miR-144- and mock-ROCK1 co-transfected SW837 and SW1463 cells at each time point. In addition, the relative proliferation rates of the miR-144 and ROCK1 co-transfected SW837 and SW1463 cells were more marked, compared with 


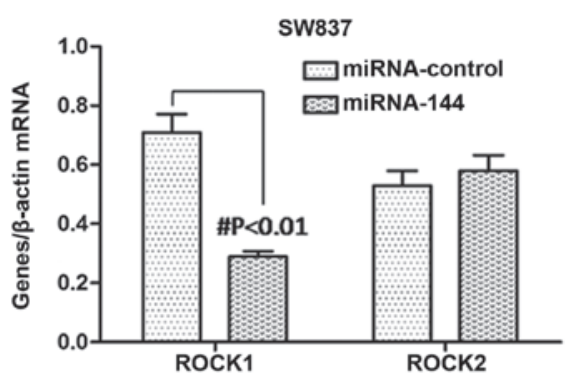

C

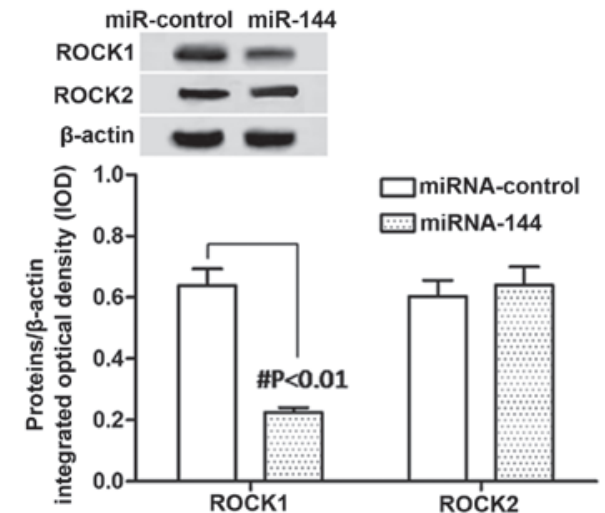

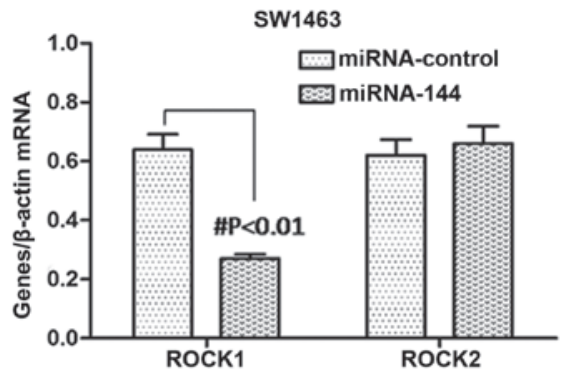

SW1463

D

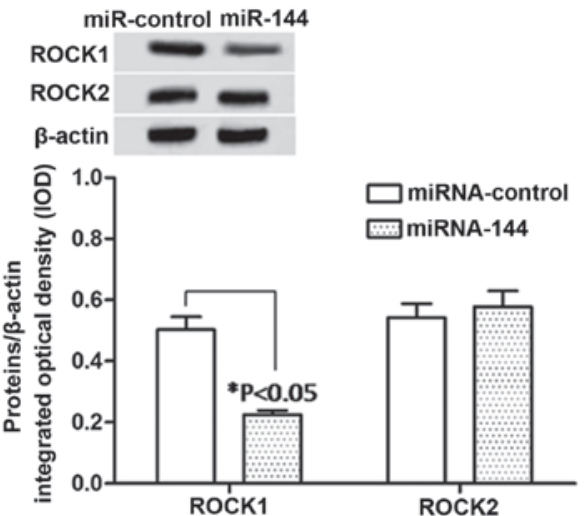

Figure 3. Expression of levels of ROCK1 and ROCK2 in miR-144-overexpressing rectal carcinoma cells (SW837 and SW1463). mRNA levels of ROCK1 and ROCK 2 mRNA were detected in the (A) SW837 and (B) SW1463 cells using reverse transcription-quantitative polymerase chain reaction following miR-144 transfection for $72 \mathrm{~h}$. Data were normalized based on the mRNA levels of $\beta$-actin. (C and D) Protein expression levels of ROCK1 and ROCK2 were detected using western blot analysis following miR-144 transfection for $72 \mathrm{~h}$. The bands on the western blot were analyzed using Gel-Pro analyzer 4.0 software. Data were normalized based on the levels of $\beta$-actin. Each data point was obtained from three repeated experiments and expressed as the mean \pm standard deviation. ${ }^{\#} \mathrm{P}<0.01$ and ${ }^{*} \mathrm{P}<0.05$ (Student's $t$-test). miR, microRNA; ROCK, Rho-associated coiled-coil containing protein kinase.

A

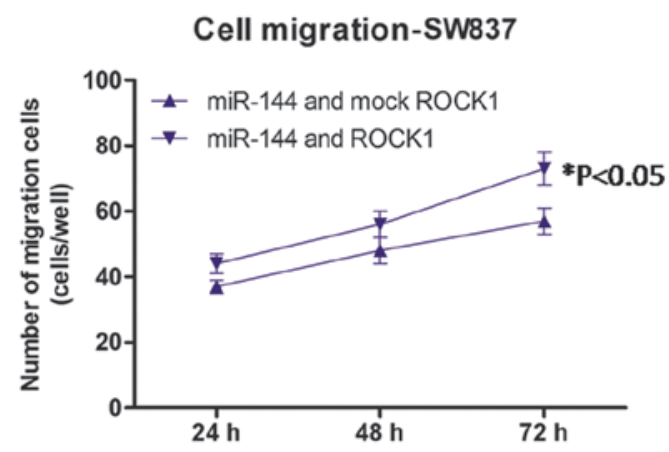

$\mathbf{C}$

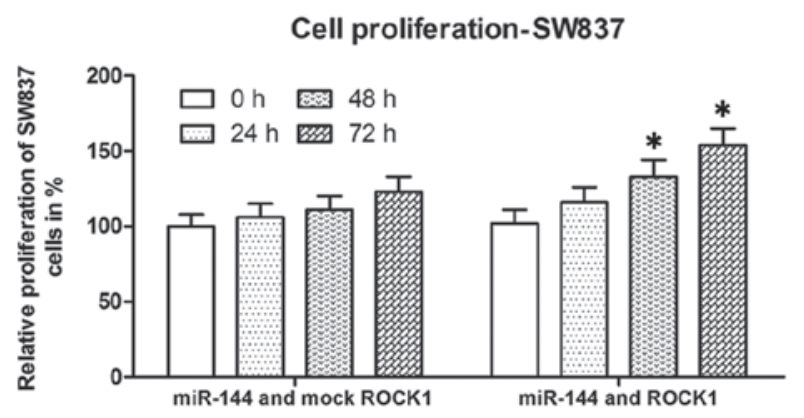

B

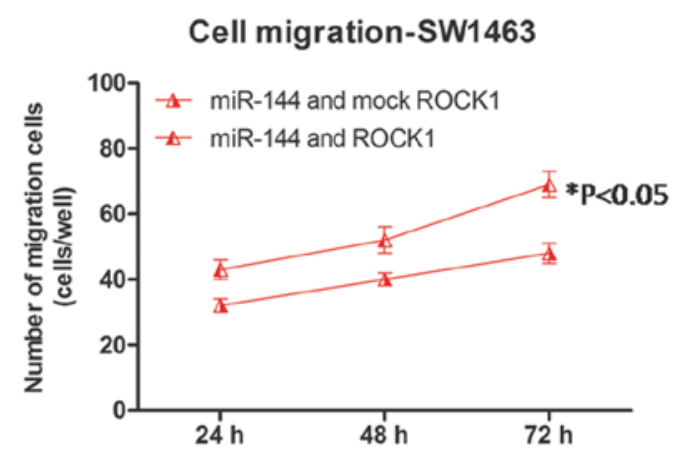

D

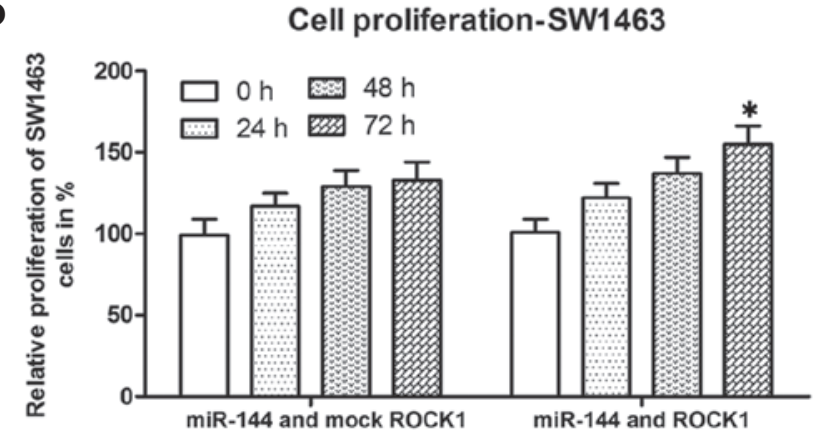

Figure 4. Cell migration and proliferation in miR-144 and ROCK1-co-transfected rectal carcinoma cells (SW837 and SW1463). Numbers of (A) SW837 and (B) SW1463 cells migrating into the lower wells. Data were obtained 24, 48 and $72 \mathrm{~h}$ following transfection. "P<0.05, compared with the corresponding miR-144 and mock ROCK1-co-transfected group. Relative percentages of proliferation of the (C) SW837 and (D) SW1463 cells. Data were obtained 0, 24, 48 and $72 \mathrm{~h}$ following transfection. "P $<0.05$, compared with the miR-144 and mock ROCK1-co-transfected group at the corresponding time point. All data are presented as the mean \pm standard deviation of three independent experiments. miR, microRNA; ROCK, Rho-associated coiled-coil containing protein kinase. 
those of the miR-144 and mock-ROCK1 co-transfected SW837 or SW1463 cells following culture for 48 and 72 h (SW837), and $72 \mathrm{~h}$ (SW1463). These results suggested that the inhibitory effects of overexpressed miR-144 on the SW837 and SW1463 cells was by the overexpression of ROCK1. Taken together, it was concluded that miR-144 inhibited migration and proliferation in rectal cancer through the downregulation of ROCK1.

\section{Discussion}

Although the expression of several miRNAs are aberrantly altered in rectal cancer (1), their underlying molecular mechanisms in the development and progression of rectal cancer remain to be fully elucidated. Thus, investigating the function of miRNAs, which are specifically involved in the progression of rectal cancer is required to improve current knowledge of rectal cancer, and offer novel insights into its diagnosis and therapy.

The present study focused on the role of miR-144 in rectal cancer, which has been extensively investigated and reported in other types of cancer, including lung cancer (25), prostate cancer (26) and hepatocellular carcinoma (27). Previous microRNA microarray analyses have indicated that miR-144 shows aberrant expression and appears to be specific to rectal cancer (1). In order to determine the role of miR-144 in rectal cancer, the present study selected SW837 and SW1463 cells as rectal cell carcinoma cells, which originated from rectal tissue belonging to epithelial cells. The results demonstrated that the expression of miR-144 was significantly reduced in rectal cell carcinoma cells, and overexpression of miR-144 repressed the viability, migration and proliferation of the SW837 and SW1463 cells in vitro by targeting ROCK1.

Previously, miR-144 was identified as an erythroid-specific miRNA, which is essential for the subsequent maturation and survival of the erythroid lineage $(12,28)$. miR-144 can decrease glutathione regeneration and antioxidant capacity by directly regulating a central regulator of the cellular response to oxidative stress (29). In addition, a previous study showed that miR-144 can increase cell growth in HeLa cells (30). Zhao et al reported that the downregulation of miR-144 is associated with the growth and invasion of osteosarcoma cells through the regulation of the expression of TAGLN (31). Cao et al reported that miR-144 suppresses the proliferation and metastasis of hepatocellular carcinoma by targeting E2F transcription factor 3 (E2F3) (27), and Guan et al reported that the downregulation of miR-144 promotes thyroid cancer cell invasion by targeting zinc finger E box binding homeobox (ZEB)1 and ZEB2 (32). In the present study, ROCK1 was identified as a novel target of miR-144, by which miR-144 inhibited the migration and proliferation of rectal cancer cells.

ROCK1 is one of the members of the ROCK family, which facilitates reorganization of the actin cytoskeleton during motion (33). Previous studies have demonstrated that ROCK1 functions as an oncogene and possesses a wide range of functions, including invasion, migration and metastasis (34-37). The expression of ROCK1 has also been found to be increased in several types of cancer, including glioma, prostate cancer, osteosarcoma and gastric cancer $(38,39)$, and ROCK1 is targeted by several miRNAs, including miR-584 (40), miR-340 (39), and miR-124 (41). In the present study, ROCK1 was identified as a novel target of miR-144 in rectal cancer cells. ROCK1 was significantly downregulated in miR-144-overexpressing SW837 and SW1463 cells. In addition, the inhibitory effects on the migration and proliferation of the miR-144 on SW837 and SW1463 cells was controlled by the overexpression of ROCK1.

In conclusion, the present study demonstrated that miR-144 suppressed the progression of rectal cancer by targeting ROCK1, suggesting that miR-144 may be a novel biomarker and therapeutic target for rectal cancer treatment.

\section{References}

1. Gaedcke J, Grade M, Camps J, Søkilde R, Kaczkowski B, Schetter AJ, Difilippantonio MJ, Harris CC, Ghadimi BM, Møller S, et al: The rectal cancer microRNAome-microRNA expression in rectal cancer and matched normal mucosa. Clin Cancer Res 18: 4919-4930, 2012.

2. Slattery ML, Wolff E, Hoffman MD, Pellatt DF, Milash B and Wolff RK: MicroRNAs and colon and rectal cancer: Differential expression by tumor location and subtype. Gene Chromosomes Cancer 50: 196-206, 2011

3. Smalheiser NR and Torvik VI: A population-based statistical approach identifies parameters characteristic of human microRNA-mRNA interactions. BMC Bioinformatics 5: 139, 2004.

4. Li P, He QY and Luo CQ: Overexpression of miR-200b inhibits the cell proliferation and promotes apoptosis of human hypertrophic scar fibroblasts in vitro. J Dermatol 41: 903-911, 2014.

5. Zhao J, Li X, Zou M, He J, Han Y, Wu D, Yang H and Wu J: miR-135a inhibition protects A549 cells from LPS-induced apoptosis by targeting Bcl-2. Biochem Bioph Res Commun 452: 951-957, 2014.

6. Ma L, Teruya-Feldstein J and Weinberg RA: Tumour invasion and metastasis initiated by microRNA-10b in breast cancer. Nature 449: 682-688, 2007.

7. Nicoloso MS, Spizzo R, Shimizu M, Rossi S and Calin GA: MicroRNAs-the micro steering wheel of tumour metastases. Nat Rev Cancer 9: 293-302, 2009.

8. Siegel R, Naishadham D and Jemal A: Cancer statistics, 2013. CA Cancer J Clin 63: 11-30, 2013.

9. Ferlay J, Shin HR, Bray F, Forman D, Mathers C and Parkin DM: Estimates of worldwide burden of cancer in 2008: GLOBOCAN 2008. Int J Cancer 127: 2893-2917, 2010.

10. Michael MZ, O' Connor SM, van Holst Pellekaan NG, Young GP and James RJ: Reduced accumulation of specific microRNAs in colorectal neoplasia. Mol Cancer Res 1: 882-891, 2003.

11. Cancer Genome Atlas Network: Comprehensive molecular characterization of human colon and rectal cancer. Nature 487: 330-337, 2012.

12. Dore LC, Amigo JD, Dos Santos CO, Zhang Z, Gai X, Tobias JW, Yu D, Klein AM, Dorman C, Wu W, et al: A GATA-1-regulated microRNA locus essential for erythropoiesis. P Natl Acad Sci USA 105: 3333-3338, 2008.

13. Lawrie CH: microRNA expression in erythropoiesis and erythroid disorders. Brit J Haematol 150: 144-151, 2010.

14. Sangokoya C, Telen MJ and Chi JT: microRNA miR-144 modulates oxidative stress tolerance and associates with anemia severity in sickle cell disease. Blood 116: 4338-4348, 2010.

15. Wang W, Peng B, Wang D, Ma X, Jiang D, Zhao J and Yu L: Human tumor microRNA signatures derived from large-scale oligonucleotide microarray datasets. Int J Cancer 129: 1624-1634, 2011.

16. Lock FE, Ryan KR, Poulter NS, Parsons M and Hotchin NA: Differential regulation of adhesion complex turnover by ROCK1 and ROCK2. PloS One 7: e31423, 2012.

17. Schmandke A, Schmandke A and Strittmatter SM: ROCK and Rho: Biochemistry and neuronal functions of Rho-associated protein kinases. Neuroscientist 13: 454-469, 2007.

18. Madaule P, Furuyashiki T, Eda M, Bito H, Ishizaki T and Narumiya S: Citron, a Rho target that affects contractility during cytokinesis. Microsc Res Tech 49: 123-126, 2000.

19. Narumiya S, Tanji M and Ishizaki T: Rho signaling, ROCK and mDia1, in transformation, metastasis and invasion. Cancer Metast Rev 28: 65-76, 2009. 
20. Wilkinson S, Paterson HF and Marshall CJ: Cdc42-MRCK and Rho-ROCK signalling cooperate in myosin phosphorylation and cell invasion. Nat Cell Biol 7: 255-261, 2005.

21. Lin SL, Chiang A, Chang D and Ying SY: Loss of mir-146a function in hormone-refractory prostate cancer. RNA 14 417-424, 2008.

22. Simionescu N, Niculescu LS, Sanda GM, Margina D and Sima AV: Serum microRNA profiling of hyperlipidemic and/or hyperglycemic patients reveals specifically increased levels of miR-122, miR-125a, miR-486 and miR-92a. Ann Rom Soc Cell Biol 19: 53-64, 2014.

23. Zheng LH, Zhang DZ, Zhang YF, Wen YH and Wang YC: mTOR signal transduction pathways contribute to TN-C FNIII A1 overexpression by mechanical stress in osteosarcoma cells. Mol Cells 37: 118-125, 2014.

24. Han L, Liang XH, Chen LX, Bao SM and Yan ZQ: SIRT1 is highly expressed in brain metastasis tissues of non-small cell lung cancer (NSCLC) and in positive regulation of NSCLC cell migration. Int J Clin Exp Patho 6: 2357, 2013.

25. Zha W, Cao L, Shen Y and Huang M: Roles of Mir-144-ZFX pathway in growth regulation of non-small-cell lung cancer. PLoS One 8: e74175, 2013.

26. Walter BA, Valera VA, Pinto PA and Merino MJ: Comprehensive microRNA profiling of prostate cancer. J Cancer 4: 350-357, 2013.

27. Cao T, Li H, Hu Y, Ma D and Cai X: miR-144 suppresses the proliferation and metastasis of hepatocellular carcinoma by targeting E2F3. Tumor Biol 35: 10759-10764, 2014.

28. Fu YF, Du TT, Dong M, Zhu KY, Jing CB, Zhang Y, Wang L, Fan HB, Chen Y, Jin Y, et al: Mir-144 selectively regulates embryonic alplha-hemoglobin synthesis during primitive erythropoiesis. Blood 113: 1340-1349, 2009.

29. Sangokoya C, Telen MJ and Chi JT: microRNA miR-144 modulates oxidative stress tolerance and associates with anemia severity in sickle cell disease. Blood 116: 4338-4348, 2010.

30. Cheng AM, Byrom MW, Shelton J and Ford LP: Antisense inhibition of human miRNAs and indications for an involvement of miRNA in cell growth and apoptosis. Nucleic Acids Res 33: 1290-1297, 2005.
31. Zhao M, Huang J, Gui K, Xiong M, Cai G, Xu J, Wang K, Liu D, Zhang X and Yin W: The downregulation of miR-144 is associated with the growth and invasion of osteosarcoma cells through the regulation of TAGLN expression. Int J Mol Med 34: 1565-1572, 2014.

32. Guan H, Liang W, Xie Z, Li H, Liu J, Liu L, Xiu L and Li Y: Down-regulation of miR-144 promotes thyroid cancer cell invasion by targeting ZEB1 and ZEB2. Endocrine 48: 566-574, 2015.

33. Wen X, Ding L, Wang JJ, Qi M, Hammonds J, Chu H, Chen X, Hunter E and Spearman P: ROCK1 and LIM Kinase modulate retrovirus particle release and cell-cell transmission events. J Virol 88: 6906-6921, 2014.

34. Shin JY, Kim YI, Cho SJ, Lee MK, Kook MC, Lee JH, Lee SS, Ashktorab H, Smoot DT, Ryu KW, et al: MicroRNA 135a suppresses lymph node metastasis through down-regulation of ROCK1 in early gastric cancer. PLoS One 9: e85205, 2014.

35. Rath N and Olson MF: Rho-associated kinases in tumorigenesis: re-considering ROCK inhibition for cancer therapy. EMBO Rep 13: 900-908, 2012.

36. Liu X, Choy E, Hornicek FJ, Yang S, Yang C, Harmon D, Mankin $\mathrm{H}$ and Duan Z: ROCK1 as a potential therapeutic target in osteosarcoma. J Orthop Res 29: 1259-1266, 2011.

37. Vigil D, Kim TY, Plachco A, Garton AJ, Castaldo L, Pachter JA, Dong H, Chen X, Tokar B, Campbell SL, et al: ROCK1 and ROCK 2 are required for non-small cell lung cancer anchorageindependent growth and invasion. Cancer Res 72: 5338-5347, 2012.

38. Zhang C, Zhang S, Zhang Z, He J, Xu Y and Liu S: ROCK has a crucial role in regulating prostate tumor growth through interaction with c-Myc. Oncogene 33: 5582-5591, 2014.

39. Zhou X, Wei M and Wang W: MicroRNA-340 suppresses osteosarcoma tumor growth and metastasis by directly targeting ROCK1. Biochem Biophys Res Commun 437: 653-658, 2013.

40. Ueno K, Hirata H, Shahryari V, Chen Y, Zaman MS, Singh K, Tabatabai ZL, Hinoda Y and Dahiya R: Tumour suppressor microRNA-584 directly targets oncogene Rock-1 and decreases invasion ability in human clear cell renal cell carcinoma. $\mathrm{Br}$ J Cancer 104: 308-315, 2011.

41. Hu CB, Li QL, Hu JF, Zhang Q, Xie JP and Deng L: miR-124 inhibits growth and invasion of gastric cancer by targeting ROCK1. Asian Pac J Cancer Prev 15: 6543-6546, 2014. 